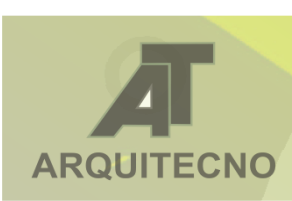

\title{
MAPER: NUEVOS MATERIALES CON PLÁSTICOS DE RSU SIN RECICLAJE
}

Eje 3: Extensión universitaria y transferencia tecnológica

\author{
Wadel Gerardo ${ }^{1}$ \\ Pérez Angueira Luciana² \\ Giacomelli Fernando3 \\ Scheffer Andrea ${ }^{4}$ \\ Pascual Macías Germana 5
}

\begin{abstract}
${ }^{1}$ Fac. Reg. Trenque Lauquen, Univ. Tecnológica Nacional, Argentina, gerardo.wadel@gmail.com
${ }^{2}$ Fac. Reg. Trenque Lauquen, Univ. Tecnológica Nacional, Argentina, lucianaperezangueira@hotmail.com

${ }^{3}$ Fac. Reg. Trenque Lauquen, Univ. Tecnológica Nacional, Argentina, fernandogiacomelli@hotmail.com

${ }^{4}$ Fac. Reg. Trenque Lauquen, Univ. Tecnológica Nacional, Argentina, andrea.scheffer@hotmail.com

${ }^{5}$ Dir. Prov. de Pol. Socio Educativa, DGCyE, Pcia. de Buenos Aires, Argentina, g.pascualmacias@hotmail.com
\end{abstract}

\section{RESUMEN}

MAPER es un proyecto de investigación y desarrollo de la Secretaría de Ciencia, Tecnología y Posgrado de la Universidad Tecnológica Nacional, con sede en la Facultad Regional Trenque Lauquen, Argentina. Su objetivo es la determinación de un modelo de gestión para el reciclaje de la fracción de plástico film y otros provenientes de los Residuos Sólidos Urbanos que actualmente no se recuperan, sino que se entierran o incineran en la mayoría de los municipios argentinos. Este modelo de gestión está siendo plasmado en una guía de implantación de la metodología de separación, recogida, tratamiento previo, proceso de reciclaje, obtención del material resultante y utilidades del mismo una vez aplicado, de carácter gratuito y uso libre para los municipios y otras entidades del sector público. Se han cuantificado las cantidades de este residuo, estudiado las tecnologías de reciclaje, seleccionado las más adecuadas, construido máquinas de reciclado a escala demostrativa, realizado pruebas de fabricación del nuevo material y desarrollado algunas posibilidades de aplicación, tanto en equipamiento urbano como en construcción de edificios. Para ello, el equipo de investigación ha contado con la colaboración de la Municipalidad de Trenque Lauquen, la Facultad de Arquitectura y Urbanismo de la Universidad Nacional de La Plata y las empresas argentina Ewar y españolas Zicla y Solteco, especializadas en la fabricación de materiales nuevos a partir de residuos industriales y domésticos. Los resultados alcanzados y las estimaciones realizadas, en momentos en que la investigación está llegando a su fin, permiten afirmar que es económica y técnicamente viable la reutilización de estos residuos hasta ahora no 
valorizados y su conversión en un material aplicable a las funciones de solado, separación, mobiliario, protección solar y construcciones abiertas con lugar en espacios abiertos y exteriores de edificios.

\section{PALABRAS CLAVE: RESIDUOS SÓLIDOS URBANOS - RECICLAJE - PLÁSTICO - TECNOLOGÍA, PRODUCCIÓN}

\section{INTRODUCCIÓN}

\subsection{El problema global del residuo plástico}

Una de las formas más sencillas de comprender rápidamente la gravedad y la magnitud del problema del uso creciente de plástico en ciclo material abierto, esto es extrayendo materias primas no renovables y vertiendo residuos contaminantes, es observando lo que ocurre en los océanos. EI UNEP, Programa Ambiental de las Naciones Unidas, calculó ya en 2006 que por cada milla cuadrada del Océano Pacífico había 46.000 piezas de plástico. Algunas estimaciones hablan de ocho millones de toneladas de plástico al año, lo que equivale a un camión de basura lleno por minuto, que acaban en el mar. Otras especies y los seres humanos, bebemos y comemos microplásticos que encontramos en todas las criaturas vivas, principalmente residuos de tereftalato de polietileno, polipropileno y polietileno, que son imposibles de destruir para los microorganismos, permaneciendo sin degradarse durante cientos de años.

El problema continúa si se mira en tierre firme: contaminación del aire por incineración o del suelo y el agua por su enterramiento (América del Sur), obturación de drenajes e inundación de campos de cría de ganado o cultivos de alimentos (África), ingesta y muerte por contaminación o inanición posterior de diversas especies de animales (Asia). Esto ha llevado a la aplicación de distintas políticas para paliar el uso y vertido de diversos productos de consumo masivo fabricados en plástico, como bolsas de compra, embajales diversos, vajilla de usar y tirar, elementos de limpieza personal, etc. Por ejemplo, en Argentina se han prohibido las bolsas de compra general en supermercados, en Marruecos se impide su fabricación o comercialización, en otros países africanos no es posible utilizar ni transportar embalajes plásticos y en España, a pesar de diversas acciones que fomentan la eliminación del plástico en usos efímeros, las cantidades finales no bajan porque, por ejemplo, mientras desaparecen las bolsas grandes aumentan los embalajes de los productos.

Se imponen otras acciones complementarias. Primero, para mejorar la salud e impedir que los plásticos sigan llegando al agua y a la cadena alimentaria, así como contaminando el medio y consumiendo recursos no renovables. Y, segundo, para fomentar la economía circular. La industria y la gestión pública pueden tener un papel de líder en nuevas tecnologías y materiales, disminuyendo la extracción de materias primas no renovables y la emisión de residuos contaminantes.

\subsection{El problema municipal del residuo plástico}

La ciudad de Trenque Lauquen gestiona sus residuos sólidos urbanos directamente, a través de su municipio y mediante el Programa PROLIM, pionero en concientización social, así como en la eficiencia en la separación, compostaje y reciclaje. Gran parte de los residuos inorgánicos (papel y cartón, vidrio, plásticos varios, metales, etc.) consigue ser valorizada mediante su recuperación y 
venta. Otro tanto ocurre con los residuos orgánicos, reconvertidos en tierras de abono. Todo ello se realiza en instalaciones municipales y con personal propio. Una de las fracciones que, si bien se consigue separar, no ha sido posible reutilizar hasta ahora es el polietileno de baja densidad (PEBD) y otros plásticos, proveniente mayormente de bolsas y films de embalajes. Estos residuos (Figs. 1 y 2), mezclados con restos orgánicos, presentan un elevado volumen y durante un tiempo se acopiaban, por lo que existe un cierto stock.

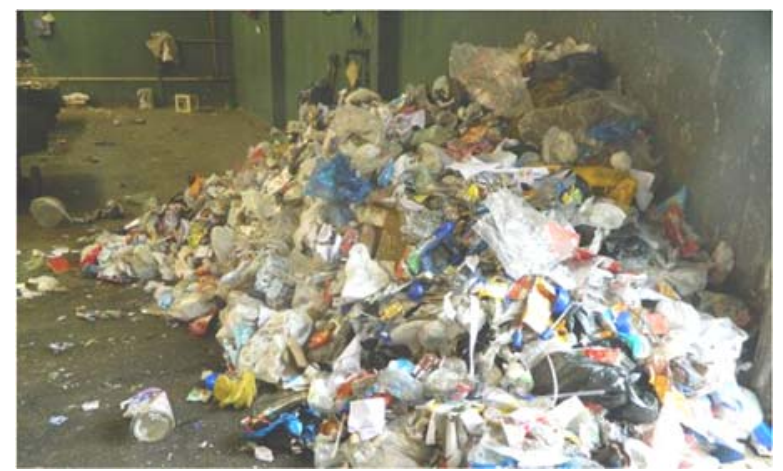

Fig. 1: PROLIM, separación de la fracción plástico film

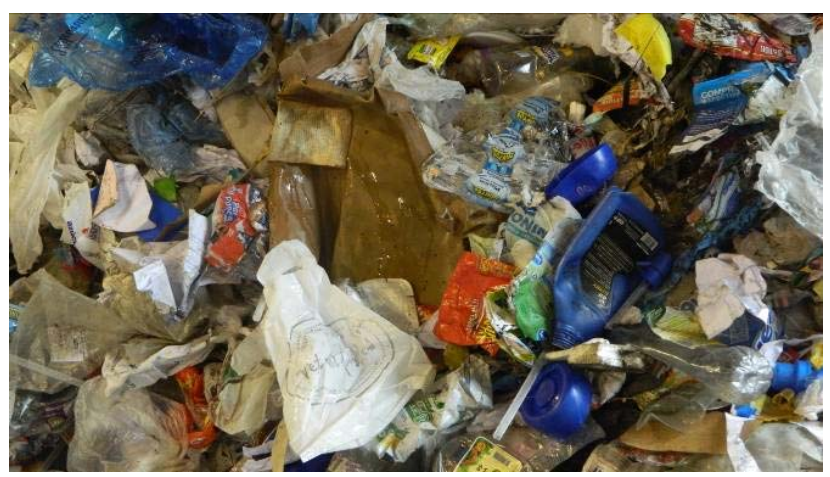

Fig 2: PROLIM, detalle de la fracción plástico film

Actualmente son enterrados prácticamente sin tratamiento ni protección del suelo. Se trata de materiales con un flujo de generación que se prevé constante y que presenta dos problemas: no se reaprovecha y contamina el medio. La situación de Trenque Lauquen se repite en otros municipios de la zona ( 9 de Julio, Bolívar, etc.) así como en el resto del país.

\subsection{Una propuesta de cambio sobre cómo actuar}

El polietileno de baja densidad, aun conteniendo residuos orgánicos y restos de otros plásticos, puede reciclarse en un material de menor calidad técnica, un tipo de plástico heterogéneo y de bajas prestaciones respecto de la materia que le da origen, aplicables a usos que no supongan elevadas calidades sanitarias, mecánicas, térmicas, etc. (figs. 3 y 4 ).

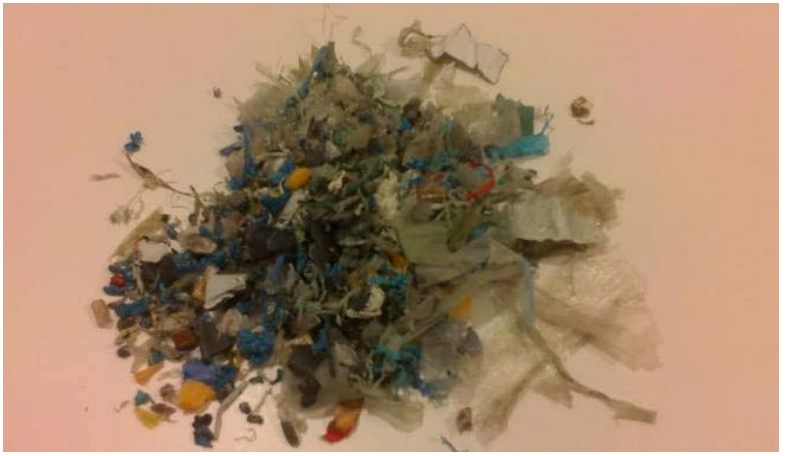

Fig. 3: Triturado de restos de polietileno (imagen Zicla)

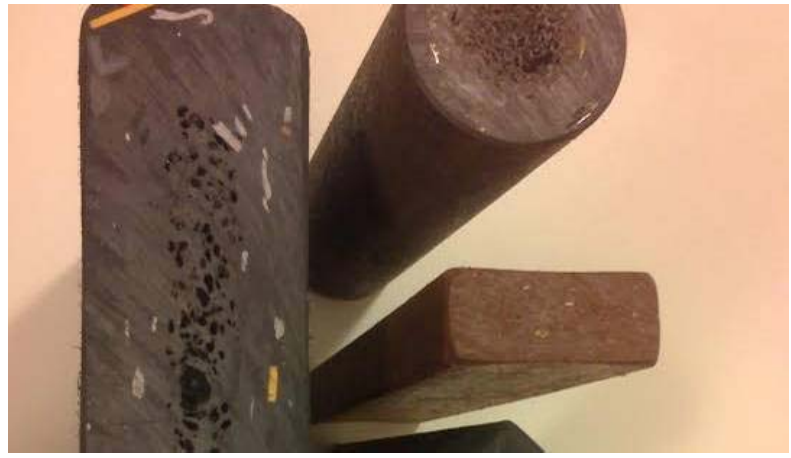

Fig 4: Perfiles de polietileno reciclado

Este plástico reciclado se obtiene a partir de la fracción de los residuos sólidos urbanos conformada por bolsas y films, que, una vez separados de otros materiales y lavados, son fundidas para obtener un primer compuesto de plásticos varios aglomerados que, posteriormente, dará lugar a una nueva fusión de perfiles o listones aplicables, por ejemplo, a pavimentos y mobiliario urbano no sometidos a exigencias técnicas importantes. Dada esta experiencia, se aprovecha el conocimiento y tecnología existentes, tanto a nivel nacional como internacional, para 
desarrollar en Trenque Lauquen y en el marco del PROLIM, un sistema de reciclaje propio y adecuado a la realidad local, de restos de PEBD y otros plásticos que actualmente no se reciclan.

\subsection{Los proyectos universitarios MAPER y PRECO}

Actualmente, el desarrollo de la tecnología de reciclaje para residuos sólidos urbanos plásticos sin aprovechamiento y sus posiblilidades de aplicación en equipamiento urbano y construcción de edificios, se lleva adelante mediante la acción complementaria de dos iniciativas.

El primero de ellos es el Proyecto de Investigación y Desarrollo Requerido (PID-R) MAPER, Material a partir de Polietileno Reciclado (código MSRENTL0003571), financiado por la Secretaría de Ciencia, Tecnología y Posgrado de la Universidad Tecnológica Nacional y requerido por la Municipalidad de Trenque Lauquen, que lleva adelante un equipo de investigación de la Facultad Regional Trenque Lauquen integrado por los autores de este artículo.

El segundo es el Proyecto del Programa Fortalecimiento de Redes Internacionales (IX Convocatoria) PRECO, Plásticos Reciclados de Residuos Sólidos Urbanos en la Construcción (número 46-146-283), financiado por la Secretaría de Políticas Universitarias del Ministerio de Educación, que llevan adelante dcuatro instituciones universitarias. La Facultad Regional Trenque Lauquen de la U. Tecnológica Nacional, proporcionando el conocimiento técnico sobre el material, y la Facultad de Arquitectura y Urbanismo de la U. Nacional de La Plata, diseñando soluciones constructivas, por Argentina. Y la Escuela Técnica Superior de Arquitectura de La Salle de la U. Ramon Llull, evaluando la puesta en obra de las soluciones, así como la Escuela Politécnica Superior de Edificación de la U. Politécnica de Cataluña, evaluando características técnicas del material, por España.

\subsection{Gobernanza, acciones con la comunidad}

Ambos proyectos se llevan adelante no solo desde una visión técnica, sino también intentando una vinculación con la comunidad, bajo un doble objetivo. Por una parte, entender las necesidades planteadas por los vecinos (en las reuniones previas a la formulación de MAPER) y por el otro hacerlos partícipes del desarrollo futuro mediante acciones formativas, divulgativas o de consulta.

En tal sentido, se llevaron a cabo algunas acciones con escuelas de educación primaria. Con el Instituto América de la localidad de Rivadavia, por ejemplo, se realizó una charla sobre plásticos y su reciclado para alumnos de 6to grado. Hubo un intercambio de ideas con los alumnos y sus docentes y se les ofreció asesoría orientada a su participación en el programa Feria de Ciencia con un proyecto orientado al reciclado. El fortalecimiento de los lazos con la sociedad, desde el ámbito educativo, busca una mayor concientización respecto al reciclado de polietileno y otros materiles, llevando el tema, el conocimiento y los ejemplos al aula. Los niños desempeñan un rol importante en sus familias, difundiendo el conocimiento, y en la sociedad futora, como ciudadanos con otros hábitos.

Al mismo tiempo se desarrolló una encuesta orientada a investigadores, profesionales especializados y funcionarios municipales, para conocer la demanda de productos de plástico reciclado para euipamiento urbano en la ciudad de Trenque Lauquen, difundida a través de una plataforma virtual y en redes sociales. Y se entrevistó al Ing. Miguel Bulián, Secretario de 
Planeamiento, Obras y Servicios Públicos de la Municipalidad de Trenque Lauquen, quien ofreció una orientación sobre dicha demanda.

\section{DESARROLLO}

\subsection{Objetivos, metodología y plan de trabajo}

El objetivo principal es desarrollar fundamentos, especificaciones técnicas y económicas preliminares para la puesta en marcha de una línea de reciclaje de restos de plástico film de polietileno y otros polímeros obtenidos de la gestión de residuos sólidos urbanos, para la fabricación de perfiles y productos de plástico reciclado, a ser utilizados en el ámbito local. Otros objetivos son formar personal docente y de investigación, aportar una visión sistémica y holística aplicable a la gestión de residuos y reciclaje así como sus información cuantitativa, apoyar conocimiento específico al desarrollo tecnológico de un área vacante en el país, difundir el conocimiento adquirido en la comunidad, integrar a distintas instituciones de educación, gobierno y representación profesional y colaborar en la reducción de la generación de residuos en Trenque Lauquen.

Entre otros aspectos del plan de trabajo, son importantes la definición del sistema técnico de gestión de la fracción de residuos de interés y el reciclado de polietileno film y otros plásticos que actualmente se entierran, la selección de la tecnología más apropiada para separar, tratar y reciclar, la elaboración de un prototipo de material y producto a partir de ese sistema y el análisis de sensibilidad técnica y económica de la hipótesis de producción. La Municipalidad de Trenque Lauquen, institución que aporta información sobre gestión de residuos, supone también un financiamiento complementario para la fabricación de máquinas de reciclado a escala que se explica más adelante. Los colegios de arquitectos (CAPBA) e ingenieros (CIPBA) de la Provincia de Buenos Aires (distritos 7 y 3 respectivamente) avalaron el proyecto y actúan como consultores en cuanto a las probables aplicaciones del material que se espera obtener. Por otra parte, en España, se cuenta con la colaboración de las empresas Zicla como asesora en tecnología, aplicación y evaluación de materiales y productos y Solteco, como asesora en el desarrollo técnico de sistemas de reciclaje de plástico film producto de residuos sólidos urbanos, características técnicas de los materiales obtenidos y aporte de productos para análisis, pruebas y prototipos.

El plan de trabajo incluye las siguientes fases semestrales: 1) Información e hipótesis, completamiento de la información necesaria para el desarrollo del proyecto y ajuste de la hipótesis de reciclaje, elaboración de material y diseño de productos; 2) Análisis y definiciones, análisis de la información recabada en la fase anterior y definirción un anteproyecto de gestión del residuo, reciclaje y fabricación de productos; 3) Pruebas piloto y modelos, análisis del material reciclado para determinar sus características técnicas y aptitudes de uso, así como definición de los principales modelos de productos que podrían fabricarse con él; 4) Validación y revisión, análiis del anteproyecto de producción, el material a obtener y los productos que pueden fabricarse, así como revisión, reformulación y validación con sus destinatarios (el municipio y la comunidad); 5) Guía de implantación, elaboración de una guía para el desarrollo de un sistema municipal de reciclaje de plásticos tipo film (polietileno y otros); 6) Comunicación (fase transversal), establecimiento de contacto frecuente con técnicos municipales, usuarios potenciales, la comunidad y otros actores de interés para el proyecto así como difundirlo entre el público especializado y el público general. 


\subsection{Datos de partida, estudios realizados}

De acuerdo a los datos obtenidos del INDEC [4] y considerando una generación per cápita de 1,2 kg RSU por día debido a las características socioeconómicas de la ciudad; se calcula que la cantidad total aproximada de RSU recolectados a diario en la ciudad de Trenque Lauquen es de 55.500 kilos; significando más de 20.000 toneladas de RSU totales generados anualmente. La aplicación de teorías estadísticas y del método de muestreo aleatorio simple para caracterización de los RSU inorgánicos, realizada por el equipo de investigación de MAPER durante los años 2014, 2016 y 2017 (fig. 5), arroja resultados similares sobre cada tipo de desecho: papel y cartón, plásticos, envases de tetrabrik, metales (ferrosos, no ferrosos y aerosoles), vidrio, residuos textiles, residuos electrónicos y repuestos (pilas, material electrónico) y otros (desechos alimenticios, madera, corcho, pañales, poda, residuos patógenos, medicamentos, misceláneos menores a $2,54 \mathrm{~cm})$.

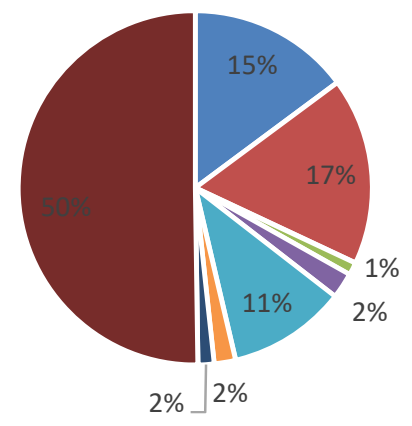

- Papel y Cartón

- Plásticos

- Envases de tetrabrik

- Metales

- Vidrio

- Residuos Textiles

- Residuos electrónicos y repuestos

- Otros

Fig. 5 Proporciones de los distintas fracciones de residuos inorgánicos en Trenque Lauquen

El total de plásticos que componen los RSU inorgánicos se clasifica en Tereftalato de polietileno (PET), Polietileno de alta densidad (PEAD), Policloruro de vinilo (PVC), Polietileno de baja densidad (PEBD), Polipropileno (PP), Poliestireno (PS) y otros. Los envases PET se separan y se clasifican por para su comercialización, mientras que el resto se envía a disposición final. Se calcula que, durante el año 2014, de forma mensual se generaron 20 toneladas de residuos plásticos no comerciales, significando un $11 \%$ del total de los RSU inorgánicos. EI PEAD constituyó aproximadamente $4 \mathrm{Tm}$, el PEBD unas 10 y el PP unas 2,5. Los otros plásticos aparecen en muy pequeña proporción. La réplica del análisis realizado en el año 2017 señala que 24 toneladas de RSU corresponden a PEAD, PEBD y PP no se comercializan, significando un $11,87 \%$ del total de los RSU inorgánicos, distribuidos de la siguiente manera: 4,5 Tm de PEAD, 10 de PEBD y 2,5 de PP. Las mediciones realizadas en los distintos años y meses, con el objetivo de evaluar la estacionalidad en la generación de RSU, aunque no se destacan resultados significativos. Considerando que la generación diaria de RSU es de 55.000 kilos se estima que un $2 \%$ de los mismos corresponden a los plásticos no comerciales de tipo PEAD, PEBD y PP que potencialmente pueden reciclarse.

\subsection{Tecnologías de reciclado, opciones más adecuadas}

El reciclado mecánico de los plásticos se utiliza casi exclusivamente para productos procedentes del consumo que han tenido una primera utilización. El procedimiento consiste en trocear el material e introducirlo en una extrusora para fabricar granza reciclada, pero cuando se trata de 
plásticos en forma de films son necesarios otros procesos. Un plástico que ha sido utilizado pierde en cierto grado sus propiedades, bien debido a la degradación que haya podido sufrir durante su uso o bien por la presencia de sustancias ajenas de los productos que contuvo. Esta merma de propiedades hace que estos plásticos reciclados deban emplearse en la fabricación en aplicaciones con menores exigencias que las del primer uso. Además, el plástico usado puede ocasionar problemas durante su transformación y no dar la calidad esperada en el nuevo producto debido a la falta de homogeneidad existen entre los mismos residuos ya que, aun tratándose del mismo tipo de plástico, pudo tener aplicaciones diferentes, como por ejemplo un polietileno procedente de un film de agricultura, de un envase de aceite o de una botella de leche. Por regla general la mezcla de plásticos diferentes, en el caso de que se puedan transformar conjuntamente dado que no todos presentan la misma estabilidad térmica, da lugar una mezcla heterogénea que no presenta buenas propiedades mecánicas para ser utilizado como material. Solamente en algunos casos las mezclas de polímeros dan lugar a una masa homogénea originando una sola fase continua, por ser los polímeros miscibles entre sí.

En el caso de MAPER, donde el material a reciclar proviene principalmente de bolsas de PEAD, se debe incorporar al proceso de reciclado mecánico una etapa de aglomeración (fig. 6). Mediante este proceso se incrementa la densidad del material a reciclar. La materia prima es introducida en la máquina aglutinadora, la cual es de forma cilíndrica, tiene cuchillas fijas en los lados y giratorias en el centro. El calor generado por la fricción de estas cuchillas eleva la temperatura del proceso y determina el incremento de la densidad del material por el encogimiento y plastificación parcial. Así, la temperatura a la que llega el proceso es a la de semiplastificación $\left(70-90^{\circ} \mathrm{C}\right)$. El material es enfriado generalmente con agua, solidificándose y tomando formas irregulares. El producto final es conocido como aglomerado, que puede pasar a una etapa de pelletizado o directamente a una de moldeo.

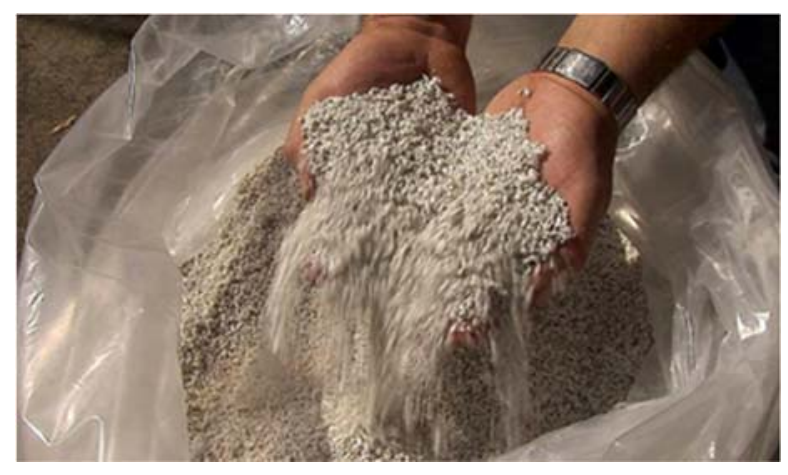

Fig. 6: Plástico reciclado aglomerado (imagen Interempresas)

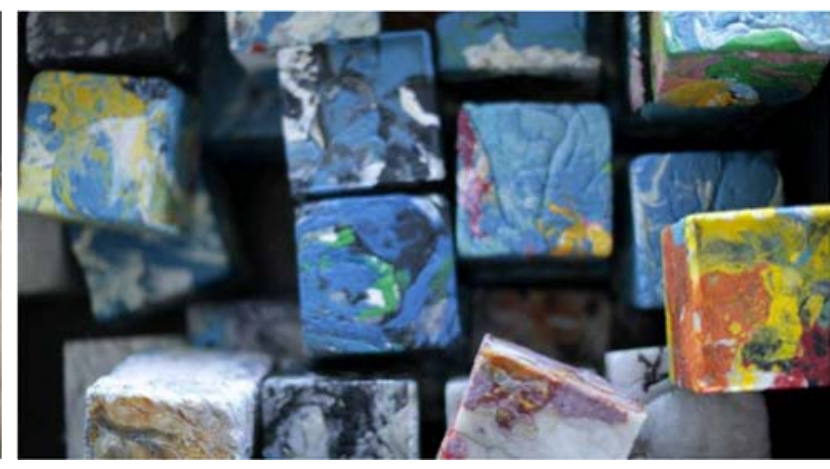

Fig 7: Plástico reciclado prensado (imagen Wasted Lab)

El material plástico reciclado (pellets, scraps, o aglomerado) puede ser procesado directamente, obteniéndose productos con menores especificaciones que las fabricadas con plástico virgen. Con la formulación adecuada, puede procesarse junto con resina virgen; esto permite una disminución en los costos de producción. Existen varias técnicas para conformar los polímeros. El termoplástico es calentado a una temperatura cercana o superior a la temperatura de fusión, de tal manera que se haga plástico o líquido. Entonces es vaciado o inyectado en un molde, produciendo la forma deseada.

Las técnicas más comunes de moldeo son inyección, soplado, extrusión, termo formado, y compresión, que es el adoptado. En el moldeo por compresión (fig. 7) el material, en general 
precalentado, es colocado en la cavidad del molde abierto. El molde se cierra, se aplica calor y presión para forzar al material a entrar en contacto con todas las áreas del molde. El moldeo por compresión es un método de alta presión, adecuado para el moldeo de piezas complejas, de alta resistencia con la posibilidad de colocar refuerzos de fibra de vidrio. Los materiales se calientan por encima de su punto de fusión, se forman y se enfrían. El material de alimentación se distribuye en forma uniforme en el molde, la orientación del flujo se produce durante la fase de compresión.

En el moldeo por compresión hay seis factores a tener en cuenta: 1) cantidad adecuada de material; 2) cantidad mínima de energía necesaria para calentarlo; 3) tiempo mínimo necesario para ese proceso; 4) técnica de calentamiento más adecuada; 5) fuerza de compresión necesaria para alcanzar la forma adecuada y 6 ) diseño de molde para un enfriamiento rápido.

En el caso de MAPER el producto a fabricar, dadas las características de la materia prima utilizada, será uno de propiedades mecánicas limitadas, y de bajo valor de reventa, por lo que se elije una técnica que requiera bajos costos de inversión inicial. Las ventajas de este proceso son: 1) fluido en pequeñas distancias: menores tensiones internas; 2) bajo costo de mantenimiento y de fabricación de moldes; 3) diseño sencillo de moldes, al no haber entrada y canales; 4) permite moldeo de piezas complejas, 5) buen acabado superficial (en general) y 6) desechos de materiales relativamente bajo.

\subsection{Máquinas para pruebas a escala y prototipos de aplicación de productos}

Con el aporte económico de la Municipalidad de Trenque Lauquen, se ha podido fabricar un juego de moldes y una máquina termoprensa (Figs. 8 y 9) para la elaboración de muestras propias, algo así como la reproducción del proceso industrial de reciclaje en laboratorio, que permitirán completar la fase experimental de reciclaje del material, atendiendo a los ajustes y calibraciones explicados en el punto anterior, así como también explicar el proceso a las instituciones educativas y la comunidad.

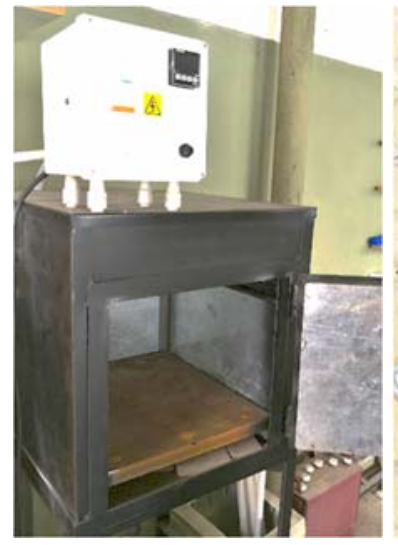

Figs. 8 y 9: Termoprensa y molde fabricados en la FRTL UTN

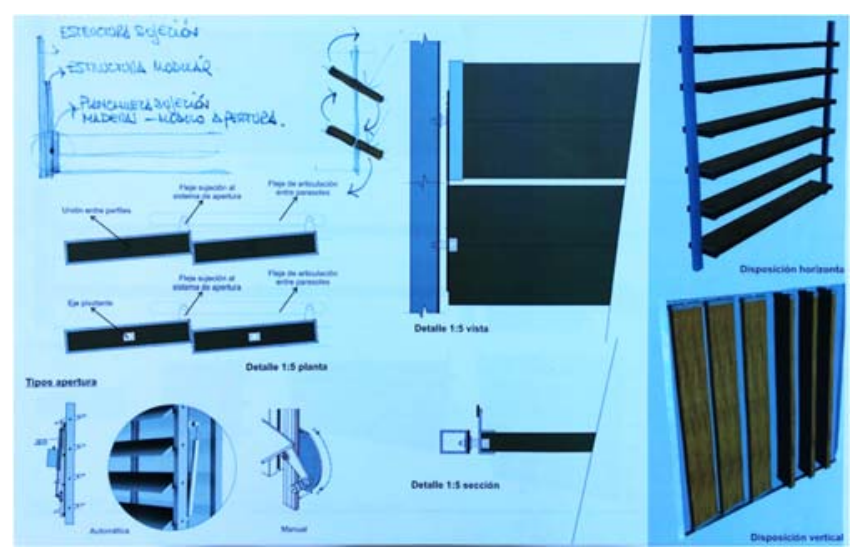

Fig 10: Parasol diseñado por alumnos de la FAU UNLP

Asimismo, con la colaboración del Taller Vertical de Procesos Constructivos No 3 LafalceLarroque-Garcia Zúñiga, de la Facultad de Arquitectura y Urbanismo de la U. Nacional de La Plata, en el marco del proyecto PRECO, se han elaborado soluciones constructivas nuevas de aplicación en construcción de edificios, como por ejemplo parasoles (Fig. 10) que se encuentran en evaluación de puesta en obra, mantenimiento y durabilidad, con la ayuda de la Cátedra de 
Organización y Control de Obras Artés-Wadel de la Escuela Técnica Superior de Arquitectura de la Universidad Ramon Llull.

\subsection{Pautas para una guía de aplicación para municipios}

La Municipalidad de Trenque Lauquen se propone rediseñar y fortalecer la Gestión Integral de Residuos Sólidos Urbanos (GIRSU) aplicando políticas, planes y programas de RSU, con una visión social y comunitaria. La separación primaria en los hogares se realiza a través de programas de concientización destinados a conseguir la colaboración de la población para la reducción de la generación y el cumplimiento de metas de valorización, reutilización y reciclado.

Frente a esa línea de trabajo se evalúan las aplicaciones industriales que pueden recibir los residuos plásticos analizados. Se consideran factores como: la preparación de la materia prima, la simpleza en el proceso productivo, las instalaciones necesarias del espacio de trabajo, los tiempos de producción, la versatilidad de aplicación, la incorporación al mercado local, la generación de puestos de trabajo, el impacto social. Por ello se considera que el proceso industrial más adecuado de implementar es la elaboración de un material reciclado en forma de perfiles y tableros, que funcione como materia prima para la elaboración productos de mayor valor agregado como: bancos y mesas para espacios libres, juegos infantiles, cestos de residuos, tarimas, maceteros, tapiales, entre otros.

La fabricación del material reciclado tiene lugar mediante un proceso limpio, que comienza con la recolección del plástico en las plantas de clasificación primaria, donde se separan los termoplásticos, luego se trituran y/o aglutinan de acuerdo al tipo de material (duros o films), se realiza un acondicionamiento simple (lavado y secado) y se procede al almacenamiento para su posterior industrialización. Los materiales deben mezclarse homogéneamente para someterse a los procesos de transformación ya descritos. Existen muchos equipos periféricos para la elaboración de productos finales, usados también para la madera y el acero. Su requerimiento depende del artículo a realizar.

De acuerdo a datos de investigaciones ya realizadas y cálculos propios, se estima que para producir un tablero de un metro cuadrado de $20 \mathrm{~mm}$ de espesor son necesarios 18,5 kilos de residuos plásticos [7]. Esto permite calcular la cantidad de tableros y perfiles potenciales a producir a partir de la cantidad de plásticos recuperados. Según cálculos propios, aproximadamente se pueden elaborar entre 1000 y 1300 metros cuadrados mensualmente. El rendimiento de los materiales semielaborados dependerá de los productos finales que se deseen fabricar.

\section{CONCLUSIONES}

- Los residuos plásticos representan la segunda porción con mayor presencia en la clasificación total de los RSU inorgánicos generados en la ciudad de Trenque Lauquen.

- Los métodos probabilísticos y de muestreo utilizados son aplicables al análisis de los RSU, dado que los datos obtenidos son similares a investigaciones realizadas por organismos certificados [8].

- Más del $60 \%$ de los residuos plásticos provenientes de los RSU inorgánicos se compone de PEAD, PEBD y PP potencialmente reciclables.

- En la actualidad se cuenta con una cantidad de residuos plásticos reciclables (PEAD, PEBD, PP) aproximada de 24 toneladas de forma mensual, lo que equivale a 288 toneladas al año; que representarían 15.500 metros cuadrados de material reciclado base para futura transformación. 
- Los residuos plásticos funcionan como una materia prima accesible, fácil de transportar y clasificar, se generan en gran cantidad y su generación se prevé más o menos constante.

- El material reciclado funciona como aislante, es insensible al agua y la humedad, no necesita protección contra insectos o bacterias, tiene una vida útil mayor a 150 años y se compone de desechos desvalorizados.

- La versatilidad del material reciclado permite fabricar infinidad de productos con una gran variedad de aplicaciones, muchas de ellas en sectores aún poco explorados como la construcción de edificios.

- Este tipo de proyectos pretende incrementar la cultura de la separación de desechos y del reciclado de materiales, mejorar las condiciones de vida de muchas familias al generar empleos, ayudar en la conformación de una sociedad mejor informada, con mayor conciencia y compromiso de mantener y mejorar el medio ambiente en el que vive.

- La metodología y la tecnología que se proponen para el reciclaje del la fracción de plásticos PEAD, PEBD y PP provenientes de RSU es aplicable a muchos otros municipios del país.

\section{BIBLIOGRAFÍA}

Abbing, M. (2018). Plastic Soup Atlas of the World Plastic Soup Foundation y LIAS publishing house. Amsterdam (Holanda) ISBN-10: 9088030960, ISBN-13: 978-9088030963.

CEAMSE. Estudio de Generación y Caracterización de los RSU. Convenio de colaboración: CEAMSE y Municipio de Trenque Lauquen" Producto №1. Trenque Lauquen, Buenos Aires, 2016.

Encuesta on-line de estimación de la demanda de productos de equipamiento urbano que podrían fabricarse con plástico reciclado en Trenque Lauquen https://es.surveymonkey.com/r/FNWH9QP

HEATmx. Elaboración de madera plástica a partir de Residuos Sólidos urbanos. Mexico, 2017. http://www.maderaplasticamx.com/madera_plastica_ficha_tecnica.html.

INDEC. Censo Nacional de Población, Hogares y Viviendas 2010. Selección realizada: Jurisdicción: Buenos Aires / Alcance geográfico: Trenque Lauquen / Unidades de relevamiento: Población / Tema: Estructura de la población. 2010.

Planta de Procesamiento (PROLIM) de residuos sólidos urbanos de la Municipalidad de Trenque Lauquen https://www.trenquelauquen.gov.ar/ecotrenque/prolim/

Santalla, L. (2013) Moldeo de plásticos, recuperado Teoría de Contrucción, en junio de 2018, en http://teoriadeconstruccion.net/blog/moldeo-de-plasticos A Coruña (España)

Taller Vertical de Procesos Constructivos No 3 Lafalce-Larroque-Garcia Zúñiga, Facultad de Arquitectura y Urbanismo, U. Nacional de La Plata https://procesosconstructivos123.wordpress.com/ 